\title{
Localization of RecA-like recombination proteins on chromosomes of the lily at various meiotic stages
}

\author{
Masahiro Terasawa, Akira Shinohara, Yasuo Hotta, ${ }^{1}$ Hideyuki Ogawa, and Tomoko Ogawa ${ }^{2}$ \\ Department of Biology, Faculty of Science, Osaka University, Toyonaka, Osaka 560, Japan; 'Department of Molecular \\ Biology, Graduate School of Bioscience, Nara Institute of Science and Technology, Ikoma, Nara 630-01, Japan
}

The Rad51 and Lim15 proteins of lily, which are homologs of the bacterial RecA protein, were found on chromosomes in various stages of meiotic prophase 1 . The presence of both Rad51 and Lim 15 proteins as discrete foci on leptotene and zygotene chromosomes and their colocalization suggest that meiotic recombination begins at the leptotene stage with the cooperation of these proteins and continues in zygotene. Localization of the foci on or adjacent to the chromosomes suggests that these proteins bind to the chromatin loops that extend from the axial cores. The proteins in these foci may participate in the searching and pairing of homologous DNA sequences, as the RecA protein does. The different pattern of localization of the Rad51 protein between the leptotene and pachytene stages and the absence of the Lim15 protein in the pachytene stage suggest that the Rad51 protein plays different roles in these stages.

[Key Words: Lily; Rad51; Lim15; meiotic recombination; chromosome synapses]

Received December 27, 1994; revised version accepted March 8, 1995

Meiotic recombination is a fundamental mechanism by which the parental traits of eukaryotes are distributed to their offspring. During the prophase of meiosis 1 , the homologous chromosomes interact and recombine with each other (Baker et al. 1976). Elaborate morphological changes occur in the chromosomes as they synapse sequentially during the leptotene, zygotene, pachytene, and diplotene stages. At the leptotene stage, each chromosome condenses from its interphase state to produce a long, thin thread with a proteinaceous central axis. Although each chromosome has replicated and consists of two sister chromatids, these chromatids are closely apposed and each chromosome therefore appears to be single. In the zygotene stage, synapsis between two homologs begins and their rope-like proteinaceous axes are brought together to form a synaptonemal complex. As soon as synapsis is complete all along the chromosomes, the cells enter the pachytene stage (for review, see von Wettstein et al. 1984). The chiasmata, which are the visible manifestations of crossover recombination, become observable in the diplotene stage.

Recombination nodules appear at intervals on the synaptonemal cmplexes of pachytene chromosomes. These nodules are thought to mediate chromosomal exchanges (Rasmussen and Holm 1978; Carpenter 1979; Zickler et al. 19921, which result in crossovers between two nonsister chromatids, one each of the two homologous chromosomes. Although invisible at pachytene, each such

${ }^{2}$ Corresponding author. crossover will appear later as a chiasma. The nodules on chromosomes found from the pachytene through diplotene stages are believed to be the sites of enzymes for meiotic recombination (Carpenter 1975, 1988; Stack et al. 1989) and are called late nodules. Another class of nodules, called early nodules, is also observed. The early nodules are abundant and have been observed along axial elements at points of convergence between homologous chromosomes, suggesting that they play a role in the initial matching of homologous regions (Albini and Jones 1987; Anderson and Stack 1988). Early nodules are considered to be the points of convergence between homologous chromosomes.

The RAD51 and RAD52 genes in Saccharomyces cerevisiae play important roles in the repair of DNA damage and in recombination (for review, see Resnick 1987; Petes et al. 1991). In meiotic recombination, they participate in repair of double-strand breaks (DSBs) formed at meiosis-specific recombination hot spots (Shinohara et al. 1992; Ogawa et al. 1993b). The domain II region of the Rad5l gene product is structurally homologous to a region of the RecA protein of Escherichia coli. This region of the Rad5l and RecA proteins is involved in formation of the nucleoprotein filament, which is crucial for the searching and pairing of the homologous sequences (Ogawa et al. 1993a,b; Sung 1994) and is required for strand transfer in genetic recombination (for review, see Kowalczykowski and Eggleston 1994). The RAD51 homologs are widely distributed in different organisms, such as ascomycete fungus, fission yeast, clawed toad, 
fruit fly, chicken, mouse, human (for review, see Kowalczykowski and Eggelston 1994; Akaboshi et al. 1994; Maeshima et al. 1995); recently a RAD51 homolog was found in a plant, Arabidopsis thaliana (K. Smith and E. Signer, pers. comm.). It seems reasonable to consider that the Rad51 homologs in different organisms perform reactions that are analogous to those played by the RecA or yeast Rad51 protein.

Genes homologous to the RAD52 gene of S. cerevisiae (Adzuma et al. 1984) are also found widely in such organisms as fission yeast, chicken, mouse, and human (Bezzubova et al. 1993; Ostermann et al. 1993; Bendixen et al. 1994; Muris et al. 1994). The purified Rad52 protein of $S$. cerevisiae carries out a strand-transfer reaction (Ogawa et al. 1993b). It has been shown that the Rad51 and Rad52 proteins of $S$. cerevisiae are involved in a species-specific interaction with each other (Shinohara et al. 1992; Milne and Weaver 1993; Ogawa et al. 1993b, 1995; Donovan et al. 1994). These results suggest that these proteins are included in a functional complex in the recombination and the repair of DNA damage.

The $D M C 1$ gene of $S$. cerevisiae is specifically expressed in meiosis. It is involved in the repair of DSBs at recombination hot spots, in the formation of synaptonemal complexes, and in the progression of the meiotic cell cycle (Bishop et al. 1992). The Dmcl protein is also structurally homologous to the Rad51 protein of $S$. cerevisiae and the RecA protein of E. coli (Story et al. 1993). The Rad51 and Dmcl proteins are required for meiotic recombination in S. cerevisiae (Bishop et al. 1992; Shinohara et al. 1992). They have been localized to the same subnuclear sites in $S$. cerevisiae, although their exact locations on chromosomes and the timing of the meiotic stages have not been determined on the yeast chromosomes. However, the requirement of Rad51 protein for binding of the Dmcl protein to the subnuclear sites suggests that these proteins may interact in the formation of a functional complex for meiotic recombination (Bishop 1994).

The LIM15 gene of the lily plant, Lilium longiflorum, is expressed specifically in prophase I of the meiotic cells (Kobayashi et al. 1993, 1994) and is a homolog of the yeast DMC1 gene (Shinohara et al. 1993). The DMC1 protein has been shown to be highly homologous to eukaryotic Rad51 proteins (Ogawa et al. 1993b). In addition to the Lim 15 protein, lily cells produce a protein that is immunologically highly homologous to the human Rad51 protein as described below. We call the protein specified by anti-human Rad51 antibody the lily Rad51 protein. Recently, both RAD51 and DMC1 genes have been cloned in Arabidopsis, suggesting that plants too have both recA-like genes (for $D M C 1$, S. Sato and S. Tabata, pers. comm.).

To understand the events that occur in each stage of meiotic recombination, it is crucial to analyze the recombination proteins present on the chromosomes in each stage. Therefore, we examined the locations of the Rad51 and Lim 15 proteins (Dmcl homolog of lily) on the chromosomes at each stage in prophase $\mathrm{I}$. We used $L$. longiflorum microsporocytes because they can be col- lected easily and the meiotic stages of their chromosomes can be identified easily. In addition, the chromosomes are large enough to analyze the localization of the Rad51 and Lim 15 proteins on the chromosomes by microscopy. Because these homologs of the RecA protein are believed to participate in the searching and pairing of homologous DNA sequences (Bishop et al. 1992; Shinohara et al. 1992; Ogawa et al. 1993a, b; Sung 1994), these processes probably occur at the sites on the chromosomes where the Rad51 and Lim 15 proteins are present.

In this report we identify the Rad51 and Lim 15 proteins as discrete foci on leptotene and zygotene chromosomes. The presence of these proteins on the chromosomes suggests that the search of homologous DNA sequences for meiotic recombination initiates on the leptotene chromosomes and leads to their pairing on the zygotene chromosomes. Colocalization of the Lim 15 and Rad51 foci on zygotene chromosomes suggests cooperation of these proteins during the early processes of recombination. Furthermore, the presence of the Rad51 protein, but not the Lim 15 protein, on pachytene chromosomes shows that the Rad51 protein also participates in later stages of meiotic recombination. This may reflect a crossover-specific function of the Rad51 protein that is not shared with Lim15.

\section{Results}

Identification of the Lim15 and Rad51 proteins in meiotic lily cells

The presence of the Lim 15 protein in different stages of meiotic cells of the lily was examined by the Western blot analysis of cell lysates using SDS-PAGE. The polyclonal antibody was made against a KLH (keyhole limpet hemocyanin) conjugate with a peptide that consists of 18 amino acid residues from the amino terminus of the Lim 15 protein (m.w. 38,270). The peptide is not homologous to any other known sequences expected for Dmcl and Rad51 homologs nor any proteins in the data bases. A single species with a mobility consistent with the size predicted by the Lim 15 coding region was found in the lysates prepared from microsporocytes in the leptotene, zygotene, and pachytene stages. This species was not detected in premeiocytes and diplotene microsporocytes (Fig. 1A). This stage, when the protein was found, coincides with the stages at which the LIM15 mRNA has been known to be present (Kobayashi et al. 1994). These results show that the protein detected by the antibody is the Lim 15 protein made in the specific meiotic stages.

Western blot analysis using the polyclonal antibody against the human Rad5l protein was used to determine the presence of the Rad51 protein in the same amount of cell lysates used for the detection of the Lim 15 protein. One protein band was abundant in the lysates prepared from premeiotic and meiotic cells (Fig. lB). Because the mobility of this band coincides with the mouse Rad51 protein used as a molecular weight marker, the protein is considered to be the Rad51 homolog (Fig. 1B, lane 1). 


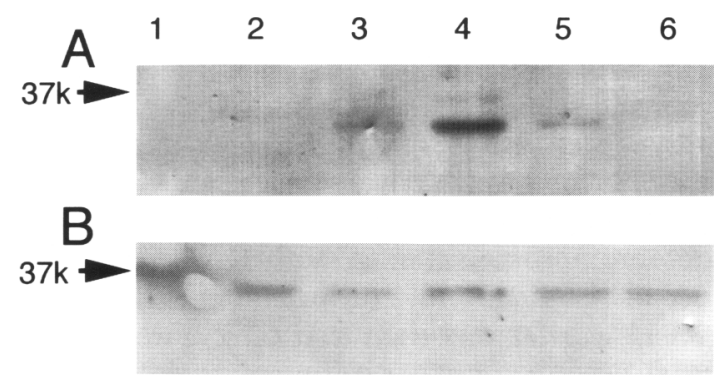

Figure 1. Western blot analysis of the Lim 15 and Rad51 proteins of premeiocytes and microsporocytes of the lily. Cell lysates were electrophoresed on a $12 \%$ SDS-polyacrylamide gel and Western blotted. Protein bands were visualized by using polyclonal rabbit anti-Lim $15 \operatorname{IgG}(A)$ or anti-Rad51 serum $(B)$. (Lane 1) A lysate prepared from 8-week-old mouse testis used as a molecular weight marker $(36,965)$; (lane 2$)$ young anthers for premeiocytes; (lanes 3-6) leptotene, zygotene, pachytene and diplotene microsporocytes, respectively.

\section{Localization of the Rad51 and Lim15 proteins on meiotic nuclei}

To examine the localization of the Rad51 or the Lim 15 protein on meiotic nuclei in the microsporocytes during the leptotene, zygotene, and pachytene stages, the nuclei spread on a glass slide were treated first with the antihuman Rad51 or anti-Lim15 antibody and then stained with FITC-labeled anti-rabbit IgG donkey serum to detect these proteins. The preparations were then stained with 4',6-diamidino-2-phenylindole (DAPI) to observe the chromosomal DNA. Observation with a UV light microscope showed that the nuclei in the premeiotic, leptotene, zygotene, and pachytene stages were stained differently with the anti-Rad51 or anti-Lim 15 antibody. In the leptotene and zygotene stages, the Rad51 and Lim15 proteins were observed as discrete foci on nuclei
[Fig. $2 \mathrm{~A}(\mathrm{a}), \mathrm{A}(\mathrm{b}), \mathrm{B}(\mathrm{a})]$. In the pachytene stage, a whole nucleus was covered by staining with the anti-Rad51 antibody and had far fewer numbers of the Rad51 foci [Fig. 2A(c)l. No staining signal with the anti-Liml5 antibody was observed on pachytene nuclei [Fig. $2 B(b)]$. Staining was observed at none of these nuclei when the preimmune serum was used (Fig. 2C). The stage-specific staining of nuclei with these antibodies probably reflects specific roles played by these proteins at different stages of meiotic recombination.

\section{Localization of the Rad51 protein on meiotic chromosomes}

To examine detailed localization of the Rad51 foci on the leptotene, zygotene, and pachytene chromosomes, the images of foci stained with the Rad51 antibody and the chromosomes stained with DAPI observed by an UV light microscope were recorded by a cooled CCD camera (Figs. 3,4,7, and 8 ). These images were then enhanced by a computer. To determine the location of Rad51 foci with respect to the chromosomes, the images of the chromosomes that were stained with the anti-Rad51 antibody were aligned with those of the same chromosomes stained with DAPI using a computer. To allow the quantitative comparison of the number of foci on the chromosomes at different stages, spreading of the chromosomes and fixing and staining of the protein were carried out under the same conditions. Although the efficiency of staining was dependent on the methods used for handling the samples, the relative numbers of foci at different stages were not changed significantly by the methods used. Therefore, we consider that the results presented here represent the values of relative abundance of the proteins on chromosomes at different stages.

Staining of meiotic chromosomes at each stage with
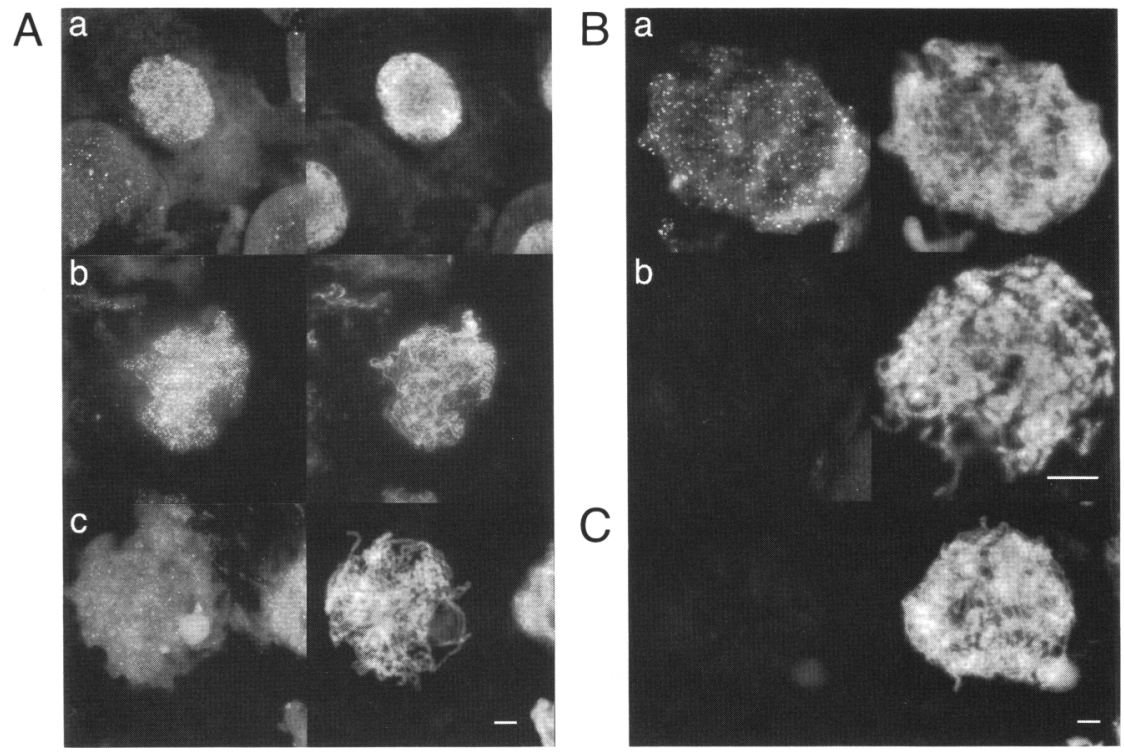

Figure 2. Immunolocalization of the Rad5l and Liml5 proteins on meiotic nuclei of the lily. The nuclei were stained with anti-human $\operatorname{Rad} 51$ antibody $(A)$, antiLim 15 antibody $(B)$, and preimmune serum for anti-human Rad51 antibody $(C)$. Photographs at left show the nuclei stained with these antibodies and then labeled with FITC-labeled anti-rabbit IgG donkey serum; those at right show nuclei stained with DAPI. Nuclei in $A$ : $(a)$ Leptotene; $(b)$ zygotene; $\{c \mid$ pachytene. Nuclei in $B:(a) \mathrm{Zy}$ gotene; $(b)$ pachytene. Nuclei in $C$ : zygotene. Bars, $10 \mu \mathrm{m}$. 
DAPI showed that the structures of chromosomes assigned as those at the zygotene, pachytene, and diplotene stages correspond to the meiotic structures of the lily assigned previously using silver staining (Stack et al. 1989). Accordingly, the chromosomes stained as thin threads and thick bands with DAPI correspond to unsynapsed and synapsed regions of chromosomes, respectively.

In the leptotene stage, chromosomes were observed as unpaired long thin threads (Fig. 3A), and many foci stained with the anti-Rad51 antibody were present (Fig. 3B). Most foci were observed along the chromosomes (Fig. 3C). In early and late zygotene, portions of chromosomes were stained thickly with DAPI, indicating that chromosomes were closely paired at these sites (Figs. $3 \mathrm{D}, \mathrm{G})$. Regions of close pairing are likely to be regions where synapsis has occurred. When the same samples were stained with the anti-Rad51 antibody, many foci were observed (Figs. 3E,H) as seen in the leptotene stage (Fig. 3B), and they were located along both the synapsed and unsynapsed regions of chromosomes (Figs. 3F,I; also see Fig. 4 , below). In the pachytene stage, synapsis was complete and the chromosomes were condensed, as seen for the chromosomes stained with DAPI (Fig. 3J). The number of the Rad51 foci on pachytene chromosomes (Fig. 3K, L) was far less than that on zygotene chromosomes, as described further below.

To show the location of the foci on the synapsed chromosomes in more detail, portions of the early zygotene chromosomes were stained with the anti-Rad5l antibody and DAPI and were photographed at a higher magnification (Fig. 4). In a region where homologous chromosomes were aligned, most foci were found on only one of homologous segments (Fig. 4A-F). The foci were
Figure 3. Immunolocalization of the Rad51 protein on meiotic chromosomes. Photographs at left $(A, D, G, J)$ show chromosomes stained with DAPI; those in the middle $(B, E, H, K)$ show chromosomes stained with anti-human Rad5I IgG; photographs at right $(C, F, I, L)$ are composite images prepared by alignment of the images of photographs at left and those in the middle. Chromosomes at right are pseudocolored in green. The spreads are from chromosomes at leptotene $(A, B$, and

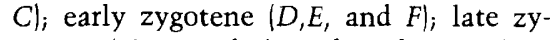
gotene $(G, H$, and $I)$; and pachytene $(I, K$, and $L$, respectively. Bars, $10 \mu \mathrm{m}$.
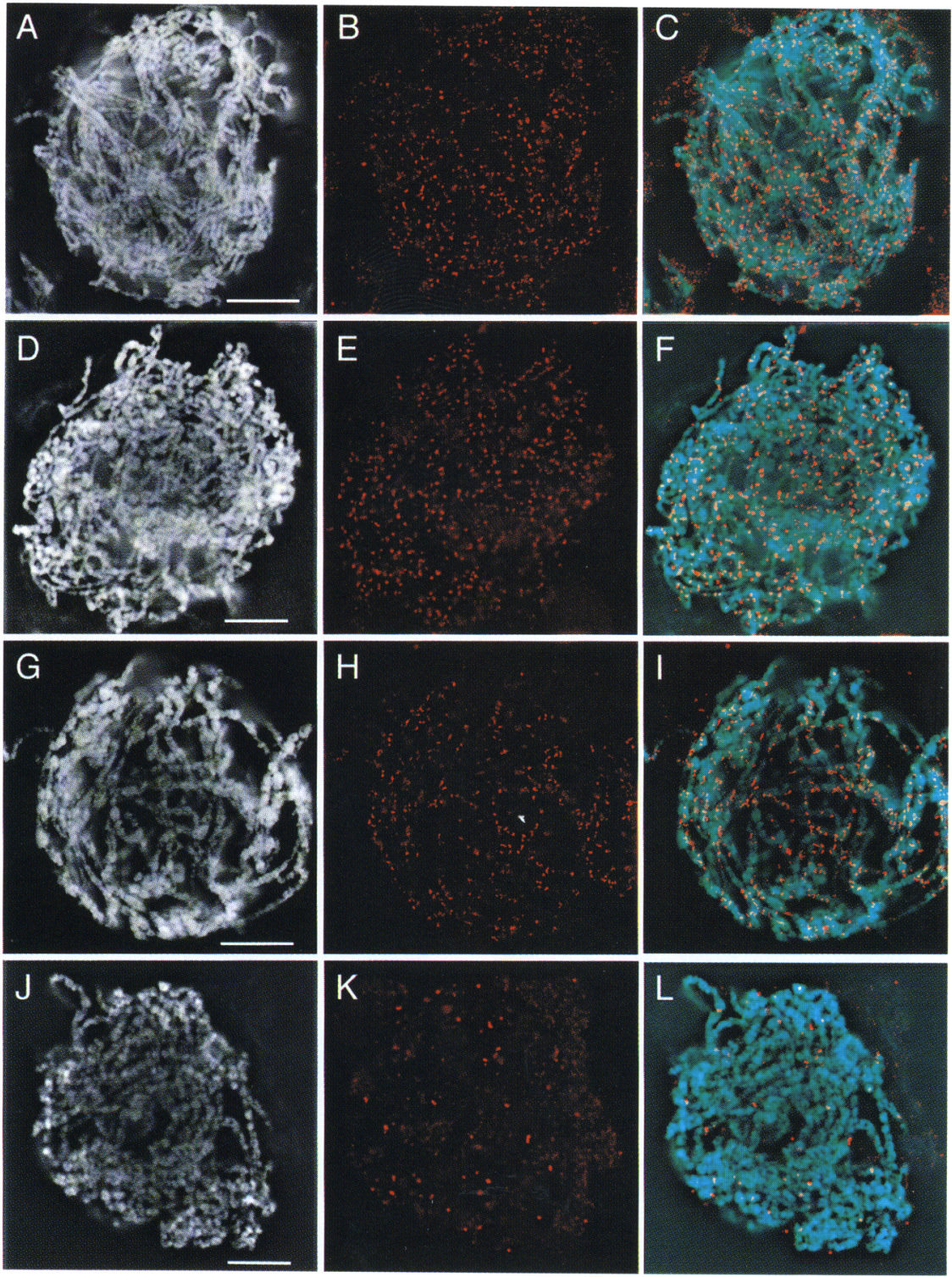

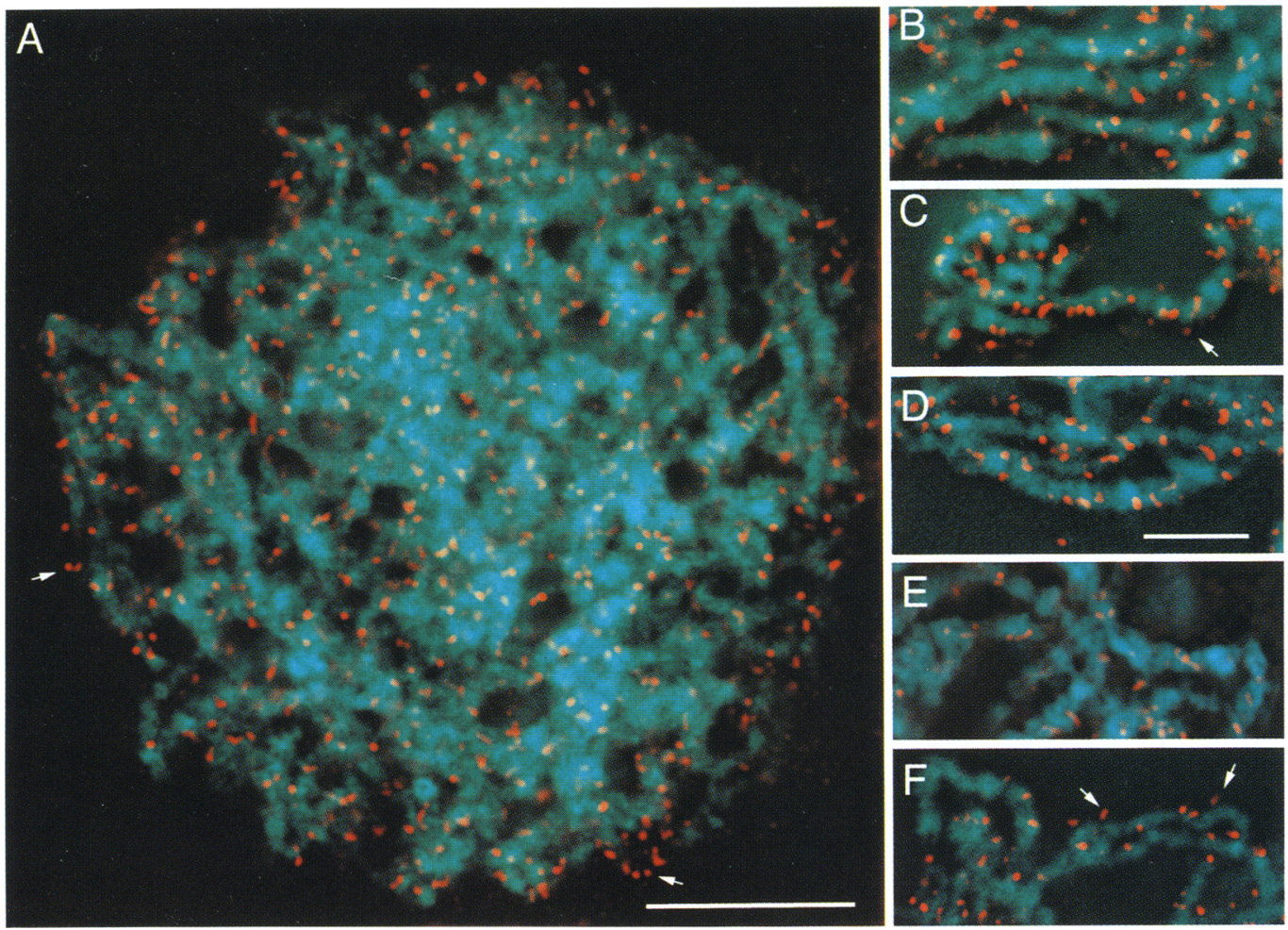

Figure 4. Location of foci stained with anti-Rad51 antibody on early zygotene chromosomes. The chromosomes were stained with the anti-Rad51 antibody and DAPI. (A) Entire spread of chromosomes with the Rad51 foci located along both the synapsed and unsynapsed regions of chromosomes. $(B-F)$ High magnification views of the foci on the chromosomes. $(B-E)$ Most foci on only one of the synapsed homologous segments. Arrows in $A, C$, and $F$ indicate examples of foci located adjacently at various distances from the chromosomes. Bars in $A$ and $D, 10$ and $5 \mu \mathrm{m}$, respectively.

frequently separated from chromosomes by varying distances (Fig. 4A,C,F). These foci may correspond to chromatin loops that extend away from the axial cores and to which the Rad51 protein bound. As shown by staining of the late zygotene chromosomes with DAPI (Fig. 3G), the homologous chromosomes are aligned almost side by side.

In the pachytene stage, the number of Rad51 foci was decreased drastically (Fig. 3K). Instead, the whole chromosomes were stained with the anti-Rad51 antibody as shown in Fig. 2A(c). To examine the pattern of localization of the Rad51 protein in the chromosomes in more detail, early pachytene chromosomes stained with the anti-Rad51 antibody were sectioned $(0.8 \mu \mathrm{m}$ interval $)$ and examined using a confocal microscope. In some regions of the sections, we found strongly stained bar-like bands in addition to closely aligned thin threads (Fig. 5). On the other hand, when the zygotene chromosomes stained with the anti-Rad5l antibody were sectioned, only foci were observed (data not shown). The structures seen in the pachytene chromosomes were not observed in the zygotene stage. The characteristic pattern of localization of the Rad51 protein on pachytene chromosomes shows the different roles of the protein during different stages in meiosis.

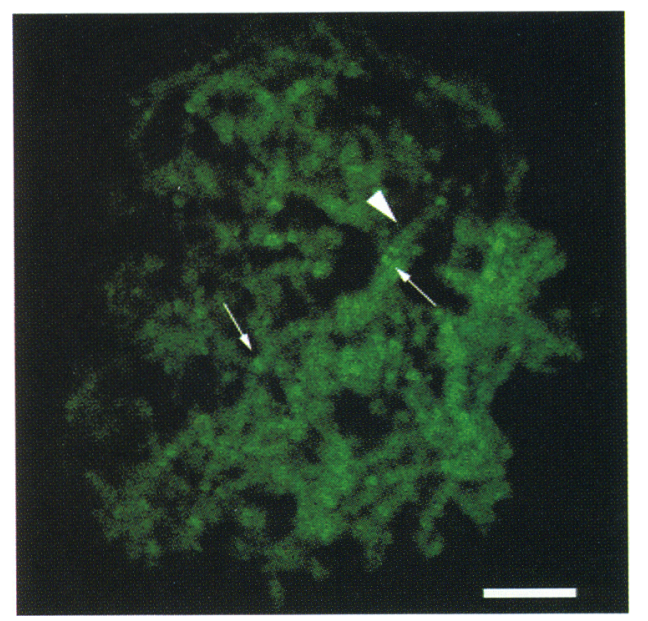

Figure 5. Localization of the Rad51 protein in a section of the early pachytene chromosomes. Staining for the Rad5l protein was carried out as described in Materials and methods. One of the sections $[0.8-\mu \mathrm{m}$ interval $\}$ of early pachytene chromosomes observed by a confocal microscopy is shown. Arrows and an arrowhead indicate examples of strongly stained bar-like bands and a thin thread-like staining, respectively. The location of chromosomes was detected by a light microscope /data not shown|. Bar, $10 \mu \mathrm{m}$. 


\section{Number of Rad51 foci on the chromosomes}

The number of foci detected by staining with the antiRad51 antibody on the chromosomes of each stage of nuclei (20-30 nuclei) was determined. The result shows that the number differed depending on the meiotic stages of the nuclei. Their average numbers are $\sim 900,600,100$, and $0 / \mathrm{mm}^{2}$ of the spread of the nucleus (size of nucleus, $-2-2.5 \mathrm{~mm}^{2}$ ) for leptotene, zygotene, pachytene, and diplotene nuclei, respectively (Fig. 6). On zygotene nuclei, the number of foci on the chromosomes decreased as the stage proceeded from early to late (see Fig. $3 \mathrm{E}, \mathrm{H}$ ).

\section{Localization of the Lim15 protein on meiotic chromosomes}

The protein on leptotene and zygotene chromosomes was stained with the anti-Lim 15 specific antibody. The Lim15 foci were present in both leptotene (data not shown) and early zygotene (Fig. 7B,C) chromosomes in a manner very similar to that of the Rad5l foci (Fig. 3E,F). The number of foci was largest in the early zygotene stage; the average of zygotene stage was $400 / \mathrm{mm}^{2}$. These results were in good agreement with the levels of proteins estimated by the Western blot analysis (Fig. 1A). To compare the pattern of localization of the Lim 15 foci on the zygotene chromosomes with that of the Rad51 foci, a part of the zygotene chromosomes was examined at a higher magnification (Fig. 7D,E). No significant difference was found in the pattern of localization of the foci between the Lim15 and Rad51 proteins in the leptotene and zygotene stages.

In the pachytene stage, no staining was observed with the anti-Lim15 antibody [Fig. $2 B(b)]$. Either the Lim 15 protein was not present on the chromosomes, or the particular epitope that was used for preparation of the antiLiml5 antibody was hidden in the structure of the pachytene chromosomes. This observation suggests the

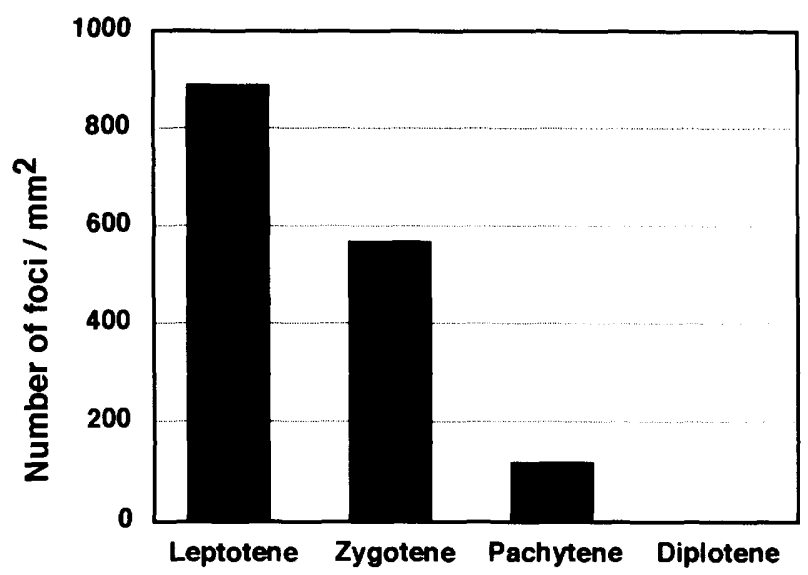

Figure 6. Number of Rad51 foci located on the chromosomes at each meiotic stage. The number of foci stained with the antiRad5l antibody in a $1 \mathrm{~mm}^{2}$ region of each nucleus were measured for leptotene, zygotene, pachytene, and diplotene nuclei.
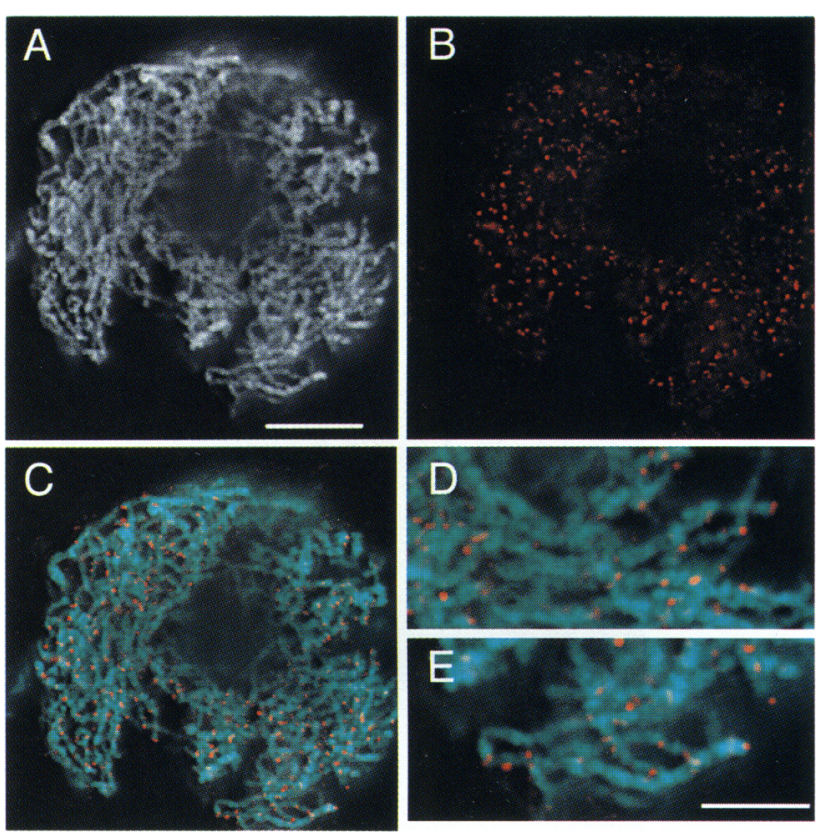

Figure 7. Localization of the Lim 15 protein on early zygotene chromosomes. The Lim 15 protein was stained with anti-Lim15specific antibody and labeled with Texas Red-labeled anti-rabbit IgG donkey serum as described in Materials and methods. $(A)$ Chromosomes stained with DAPI; $(B)$ chromosomes stained with anti-Lim15 antibody; $|C|$ a composite image of photographs in $A$ and $B$ aligned by a computer. $(D, E)$ Portions of expanded pictures of $C$. Bars in $A, B$, and $C, 10 \mu \mathrm{m}$; bars in $D$ and E, $5 \mu \mathrm{m}$.

absence or alteration of roles of the Lim 15 protein in pachytene stage.

Localization of the Rad51 and Lim15 proteins at the same sites on the chromosomes

Double staining of early zygotene chromosomes with the anti-Rad5l and anti-Lim15 antibodies was carried out to examine whether the two proteins were colocalized. The chromosomes were stained first with the antiLim 15 antibody and then labeled with Texas Red-labeled anti-rabbit IgG. Subsequently, the same sample was stained with the FITC-labeled anti-rabbit Rad51 antibody. The results show that $80 \%$ of the Rad51 and $70 \%$ of the Liml 5 foci were stained with both antibodies (Fig. $8 \mathrm{~A})$ and that many of the foci were present in the regions on or adjacent to the chromosomes (Fig. 8B).

\section{Discussion}

Using lily cells, we identified the locations of the Rad51 and Lim 15 proteins on chromosomes at different meiotic stages. The Rad51 and Lim 15 proteins were abundant on leptotene and zygotene chromosomes, forming discrete foci. The number of foci of these proteins decreased drastically as the meiotic stage proceeded from early zygotene to pachytene. A large fraction of the Rad51 and 

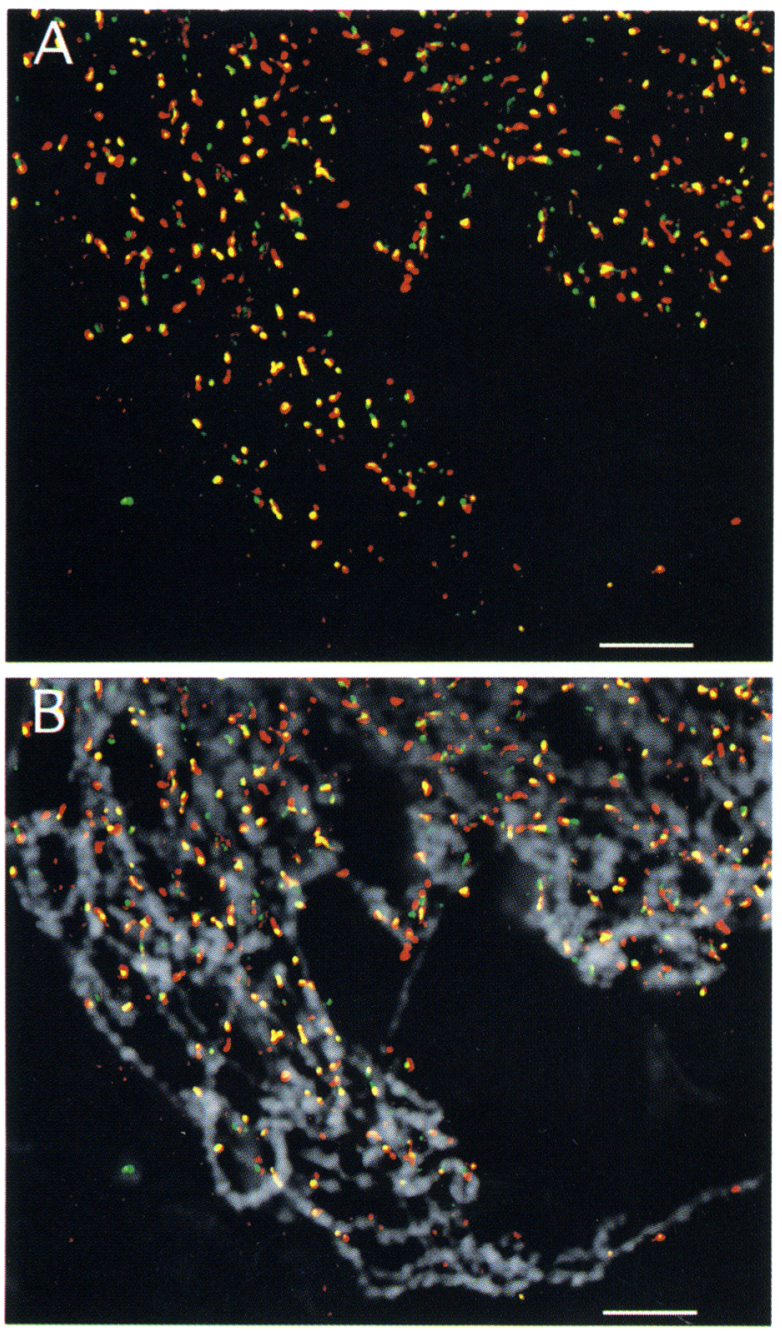

Figure 8. Colocalization of Rad5l and Lim 15 proteins. The colocalization of the Rad51 protein with Lim15 proteins on the early zygotene chromosomes was examined by concurrent staining of the chromosomes with both the anti-Liml5 and anti-Rad51 antibodies. $(A, B)$ The chromosomes were stained first with the anti-Liml $5 \mathrm{IgG}$; then, the same samples were stained further with FITC-labeled anti-rabbit Rad5I IgG as described in Materials and methods. $(B)$ The chromosomal DNA was stained further with DAPI. Red foci represent those stained with the anti-Lim 15 antibody; green foci with the anti-Rad51 antibody. Colocalization of the red and green signals yields a yellow signal. Bars, $5 \mu \mathrm{m}$.

Lim 15 foci colocalized, suggesting that those proteins may act together by forming a protein complex. Although the number decreased, a significant number of the Rad51 foci was still detected on the pachytene chromosomes, whereas no Lim 15 foci were found. Examination of Rad51 protein localization using a confocal microscope showed that the Rad51 protein is present as strongly stained bands as well as two thin thread-like structures. It is evident that the pattern of localization of the $\operatorname{Rad} 51$ protein differs as the chromosome structure is changed. These results suggest different roles for this protein during different stages of meiotic recombination.

Although the presence of homologs of the Rad51 and Lim 15 proteins in yeast subnuclear sites has recently been shown (Bishop 1994), neither their exact chromosomal localization nor the meiotic stage of chromosomes to which they bind could be identified in the yeast system. The results summarized above show that, by taking advantage of the excellence of the cytological properties of the lily system, we could significantly advance our understanding of the process of meiotic recombination.

\section{Involvement of the Rad51 protein in early process in meiotic recombination}

It has been known that $S$. cerevisiae and human Rad51 proteins form a right-handed helical nucleoprotein filament similar to that formed by the RecA protein (Ogawa et al. 1993a; Benson et al. 1994) and that the S. cerevisiae protein has strand-transfer activity (Sung 1994). We think it reasonable to consider that the Rad51 protein of the lily carries out an analogous reaction during meiotic recombination. The Rad51 protein in the recombination complexes probably searches homologous DNA sequences and initiates their pairing in a manner similar to the presynaptic complexes formed by RecA and yeast Rad51 proteins. The Rad51 foci were rarely found on the corresponding regions of both homologous chromosomes. This finding supports the hypothesis that the formation of presynaptic complexes on only one of the homologous sequences is sufficient for searching of the homologous sequences that leads to their pairing, as is the case for the homology search by the RecA protein.

The presence of Rad51 on or adjacent to the DAPIstained chromosomes suggests that the $\operatorname{Rad} 51$ protein binds to the DNA regions of the looped chromatin extended away from the axial cores of the chromosomes. On the basis of results of the three-dimensional reconstruction of a serial section of early zygotene chromosomes of the lily at the level of electron microscopy, it has been proposed that the synapsis of homologous chromosomes initiates at various places of the chromosomes and that a part of synaptonemal complex forms by attachment of the axial component after association of chromatin to the central region (Holm 1977). From these views, it is likely that the Rad51 and Lim15 foci on leptotene and zygotene chromosomes correspond to the site of initiation of recombination that begins at a stage before formation of synaptonemal complexes. These foci may correspond to early recombination nodules that have been observed along axial elements at points of convergence between homologous chromosomes.

\section{Involvement of the Rad51 protein in the late process of meiotic recombination}

On the pachytene chromosomes, the Rad51 protein was observed by confocal microscopy as strongly stained 
bands as well as two thin thread-like structures. Formation of this thin thread-like elongated structure along the chromosomes suggests that the Rad51 protein becomes a part of components of the lateral elements in synaptonemal complexes. The strongly stained bands may correspond to late recombination nodules, which have been implicated in the formation of chromosomal crossovers as precursors of chiasmata. The difference in the existence of the Rad51 protein in the early and late nodules and apparent absence of the Lim15 protein on the pachytene chromosomes show that these nodules found in different stages may have different roles in the process of meiotic recombination.

We have shown the lily to be an excellent organism for studying the localization of the Rad51 and Lim 15 proteins on meiotic chromosomes. The stage-specific colocalization of these proteins demonstrates the collaboration of these proteins in the formation of a functional structure. Formation of another stage-specific structure involving the Rad51 protein demonstrates a unique role played by the protein in a late process of meiotic recombination. In light of these results, our approach should be valuable for understanding behavior of various proteins in meiotic recombination.

\section{Materials and methods}

Preparation of antibodies

Anti-human Rad51 antibody The human Rad51 protein was overproduced in E. coli using the T7 promoter system (Studier et al. 1990). By using site-directed mutagenesis (Kunkel 1985), a $\mathrm{NcoI}$ or BamHI site was created in the first ATG site of the human Rad51 open reading frame (ORF) or just downstream of the ORF, respectively. Primers used are AGGAGCGCTGTGGACGGATCCATGGCAATGCAGATG for the NcoI site and AGACTGAAGGATCCGGTTTTTCCTCTG for the BamHI site. Plasmid pET-HsRad51 was constructed by cloning a NcoI$B a m H I$ fragment containing the human Rad5l ORF into the $N$ coI-BamHI site of pET8a plasmid (Studier et al. 1990). After $E$. coli BL21(DE3) strain (Studier et al. 1990), harboring both pETHsRad51 and pLysE plasmids, was grown to early log phase $\left(1 \times 10^{8}\right.$ cells $\left./ \mathrm{ml}\right)$ in the presence of ampicillin $(50 \mu \mathrm{g} / \mathrm{ml})$ and chloramphenicol $(20 \mu \mathrm{g} / \mathrm{ml})$, IPTG was added to the culture at a final concentration of $50 \mu \mathrm{g} / \mathrm{ml}$ and the incubation was continued for $3 \mathrm{hr}$. The cells were collected, suspended in lysis buffer [ $20 \mathrm{~mm}$ Tris- $\mathrm{HCl}$ ( $\mathrm{pH} 8.0$ ), $1 \mathrm{~mm}$ EDTA, $5 \mathrm{~mm} \beta$-mercaptoethanol, $20 \%$ (wt/vol) sucrose] and disrupted by sonication (Branson Cell Disrupter model 185). After centrifugation of the lysate at $3000 \mathrm{rpm}$, the human Rad5I protein was recovered in the pellet because it formed an inclusion body after overexpression. The pellet was washed twice with the lysis buffer and then once with buffer A [20 mM Tris- $\mathrm{HCl}\{\mathrm{pH} 8.0\}, 1$ mM EDTA, 5 $\mathrm{mM} \beta$-mercaptoethanol] containing $2 \mathrm{M}$ guanidine hydrochloride (GuHCl). The human Rad51 protein in the pellet was solubilized with buffer A containing $4 \mathrm{M} \mathrm{GuHCl}$. The solution 13.1 $\mathrm{mg} / \mathrm{ml}$ of human Rad51 protein) was used for immunizing rabbits. Purification of the IgG and labeling with FITC or Texas Red was carried out by Akagi Trading Co. (Kobe, Japan).

Anti-Lim15 antibody A peptide (MVDVKFEERRFESPGQLQ) corresponding to the 18 amino acids at the amino terminus of the Lim 15 protein was synthesized by KURABO Co. Ltd. (Osaka, Japan) and the KLH conjugate was then made and used for immunization of rabbits by Akagi Trading Co.

\section{Western blot analysis of Rad51 and Lim 15 proteins}

The anthers with premeiocytes and microsporocytes were lysed in SDS lysis buffer ( $1 \%$ SDS, $5 \% \beta$-mercaptoethanol, $30 \mathrm{~mm}$ Tris at $\mathrm{pH} 6.8$ ) containing the same volume of glass beads $(0.05$ $\mu$. diam.) with vigorous mixing using a vortex mixer. The lysates were then heated at $95^{\circ} \mathrm{C}$ for $5 \mathrm{~min}$. An aliquot $(1 \mu \mathrm{l})$ was electrophoresed in a $12 \%$ SDS-polyacrylamide gel using the Phast System (Pharmacia). The gel was stained by Coomassie brilliant blue $\mathrm{R} 250$, and the amount of protein in the designated bands was measured by a Laser Scanner (Molecular Dynamix Co. Ltd.)

The lysates containing an equal amount of protein were electrophoresed, and the gel was analyzed by Western blot using the anti-Rad51 or anti-Lim15 antibody.

\section{Preparation of nuclei and chromosomes} from microsporocytes

Microsporocytes were obtained from anthers of the lily, $L$. longiflorum, var. Hinomoto (Sakaguchi Farm, Wakayama, Japan). The meiotic stage of the cells (Erickson 1948) was determined by direct microscopic observation.

The microsporocytes were fixed in $4 \%(\mathrm{wt} / \mathrm{vol})$ paraformaldehyde for $30-40 \mathrm{~min}$ and then placed on a polylysine-coated slide glass. The cells were treated further with $0.2 \%$ Triton $\mathrm{X}-100$ for $2 \mathrm{~min}$, and a coverslip was added. The samples were squashed carefully to spread the nuclei or chromosomes and then placed on a dry-ice block. The coverslips were removed from the frozen samples, and the samples were stained with an antibody.

\section{Immunostaining}

Chromosomes were first incubated in phosphate-buffered saline (PBS; Harlow and Lane 1988 ) with $3 \%$ (wt/vol) bovine serum albumin (BSA) for $30 \mathrm{~min}$ at $23^{\circ} \mathrm{C}(200 \mu \mathrm{l}$, without a coverslip). Then, $50 \mu \mathrm{l}$ of PBS/BSA containing an appropriate dilution of a primary IgG $(1: 100)$ or the serum $(1: 2000$ for $\operatorname{Rad} 51 ; 1: 1000$ for Lim 15) was placed on polylysine-coated slides, which were incubated for $3 \mathrm{hr}$ at $23^{\circ} \mathrm{C}$. The slides were washed by dipping them three times into PBS containing $0.05 \%$ Tween 20 each for 5 min. Staining with FITC or Texas Red-labeled anti-rabbit IgG donkey serum was carried out under the same incubation conditions used for the primary antibodies except that the incubation time was $60 \mathrm{~min}$. After antibody staining, the chromosomes were stained with PBS containing $0.05 \mu \mathrm{g} / \mathrm{ml}$ of DAPI and mounted with $90 \%$ glycerol containing $1 \mathrm{mg} / \mathrm{ml}$ of $p$-phenylenediamine (antifade).

The Lim15-Rad51 sequential double staining was carried out as follows. Samples were stained with the anti-Lim 15 antibody using the procedure described above. Then, unbound anti-rabbit donkey serum was saturated with unlabeled preimmune rabbit serum and washed with PBS. The slides were then stained with FITC-labeled anti-Rad5I IgG. Slides were then washed and stained with DAPI as described above.

For primary staining of chromosomes, purified anti-Rad51 IgG or anti-Lim $15 \mathrm{IgG}$ was used after appropriate dilution. The FITC or Texas Red-labeled anti-Rad51 IgG and FITC or Texas Red-labeled anti-rabbit IgG donkey serum (Amersham) were used also after dilution. 


\section{Immunofluorescence microscopy}

Images were taken with a Nikon epiflorescence microscope (Nikon Optiphoto 2, 40× objectives with a numerical aperture of p.84 or $100 \times$ objective with NA 1.25 ) equipped with a cooled charged coupled device (CCD) camera (PXL1400-C1-M) (Photometrics), which was controlled by a Macintosh Quadra $840 \mathrm{AV}$ (Apple computer). The images were recorded as monochrome data using the CCD image capture with IP Lab-PVCAM software and Confocal-Deconvolution Software for image correction and enhancement (Signal Analysis Corporation). Merging and pseudocoloring were accomplished using Adobe Photoshop software, and the images were printed out with a Pictorography 3000 (FUJIX).

For confocal microscopy, the chromosomes were stained with anti-human Rad51 antibody and then labeled with FITC-labeled anti-rabbit IgG donkey serum. The chromosomes were located under a light microscope, and the samples were viewed under an Olympus LSM-GB 100 confocal microscope equipped with an Argon laser. Each image was analyzed using Olympus LSM-GB software.

\section{Acknowledgments}

We thank Dr. Jun-ichi Tomizawa for valuable discussions during the research and for a critical reading of the manuscript. We thank to Drs. Joseph Inselburg and Douglas Bishop for comments and Dr. Satoshi Ogihara, Mrs. Ichiro Nishii, and Masahiro Ueda for their kind help in manipulating a cooled CCD camera and confocal microscopy. This work was supported in part by a Grant-in Aid for Specially Promoted Research [06101003) from The Ministry of Education, Science, and Culture of Japan, and by a Collaboration Research grant from $\mathrm{Na}$ tional Institute of Genetics.

The publication costs of this article were defrayed in part by payment of page charges. This article must therefore be hereby marked "advertisement" in accordance with 18 USC section 1734 solely to indicate this fact.

\section{References}

Adzuma, K., T. Ogawa, and H. Ogawa. 1984. Primary structure of the RAD52 gene in Saccharomyces cerevisiae. Mol. Cell Biol. 4: 2735-2744.

Akaboshi, E., Y. Inoue, and H. Ryo. 1994. Cloning of the cDNA and genomic DNA that correspond to the recA-like gene Drosophila melanogaster. Ipn. J. Genet. 69: 663-670.

Albini, S.M. and G.H. Jones. 1987. Synaptonemal complex spreading in Allium cepa and A. fistulosum. Chromosoma 95: 324-338.

Anderson, L.K. and S.M. Stack. 1988. Nodules associated with axial cores and synaptonemal complexes during zygotene in Psilotum nudum. Chromosoma 97: 96-100.

Baker, B.S., A.T.C. Carpenter, M.S. Esposito, R.E. Esposito, and L. Sander. 1976. The genetic control of meiosis. Annu. Rev. Genet. 10: 53-134.

Bendixen, C., I. Sunjevaric, R. Bauchwitz, and R. Rothstein. 1994. Identification of a mouse homologue of the Saccharomyces cerevisiae recombination and repair gene, RAD52. Genomics 23: 300-303.

Benson, F.E., A. Stasiak, and S.C. West. 1994. Purification and characterization of the human Rad51 protein, an analog of $E$. coli RecA. EMBO J. 13: 5764-5771.

Bezzubova, O., H. Schmidt, K. Ostermann, W.D. Heyer, and J.-M. Buerstedde. 1993. Identification of a chicken RAD52 homologue suggests conversion of the RAD52 recombina- tion pathway throughout the evolution of higher eukaryotes. Nucleic Acids Res. 21: 5945-5949.

Bishop, D.K. 1994. RecA homologs Dmcl and Rad51 interact to form multiple nuclear complexes prior to meiotic chromosome synapsis. Cell 79: 1081-1092.

Bishop, D.K., D. Park, L. Xu, and N. Kleckner. 1992. DMC1: A meiosis-specific yeast homolog of $E$. coli rec $A$ required for recombination, synaptonemal complex formation, and cell cycle progression. Cell 69: 439-456.

Carpenter, A.T.C. 1975. Electron microscopy of meiosis in Drosophila melanogaster females. II. The recombination nodule-a recombination-associated structure at pachytene? Proc. Natl. Acad. Sci. 72: 3186-3189.

. 1979. Synaptonemal complex and recombination nodules in wild-type Drosophila melanogaster females. Genetics 92: 511-541.

1988. Thoughts on recombination nodules, meiotic recombination, and chiasmata. In Genetic recombination (ed. R. Kucherlaptli and G.R. Smith/ pp. 529-548. American Society for Microbiology, Washington, D.C.

Donovan, J.W., T. Milne, and D.T. Weaver. 1994. Homotypic and heterotypic protein associations control Rad51 function in double-strand break repair. Genes \& Dev. 8: 2552-2562.

Erickson, R.O. 1948. Cytological and growth correlations in the flower bud and anther of Lilium longiflorum. Am. J. Botany 35: 729-739.

Harlow, E. and D. Lane. 1988. In Antibodies: A laboratory manual. pp. 684, Cold Spring Harbor Laboratory, Cold Spring Harbor, New York.

Holm, P.B. 1977. Three-dimensional reconstruction of chromosome pairing during the zygotene stage of meiosis in Lilium longiflorum (Thunb). Carlsberg Res. Commun. 42: 103-151.

Kobayashi, T., Y. Hotta, and S. Tabata. 1993. Isolation and characterization of a yeast gene which is homologous with a meiosis specific cDNA from a plant. Mol. \& Gen. Genet. 237: 225-232.

Kobayashi, T. , E. Kobayashi, S. Sato, Y. Hotta, N. Miyajima, A. Tanaka, and S. Tabata. 1994. Characterization of cDNAs induced in meiotic prophase in lily microsporocytes. DNA Res. 1: 15-26.

Kowalczykowski, S.C. and A.K. Eggleston. 1994. Homologous pairing and DNA strand exchange proteins. Annu. Rev. Biochem. 63: 991-1034.

Kunkel, T.A. 1985. Rapid and efficient site-specific mutagenesis without phenotypic selection. Proc. Natl. Acad. Sci. 82: $488-492$.

Maeshima, K., K. Morimatsu, A. Shinohara, and T. Horii. 1995. RAD51 homologues in Xenopus laevis: Two distinct genes are highly expressed in ovary and testis. Gene 158: (in press).

Milne, G.T. and D.T. Weaver, 1993. Dominant negative alleles of $R A D 52$ reveal a DNA repair/recombination complex including Rad51 and Rad52. Gene \& Dev. 9: 1755-1765.

Muris, D.F.R., O. Benzzubova, J.-M. Buerstedde, K. Vreeken, A.S. Balajee, C.J. Osgood, C. Troelstra, J.H.J. Hoeijmarkers, K. Ostermann, H. Schmidt, A.T. Natarajan, J.C.J. Eeken, P.H.M. Lohman, and A. Pastink. 1994. Cloning of human and mouse genes homologous to RAD52, a yeast gene involved in DNA repair and recombination. Mutat. Res. 315: 295-305.

Ogawa, T., X. Yu, A. Shinohara, and E.H. Egelman. 1993a. Similarity of the yeast Rad51 filament to the bacterial RecA filament. Science 259: 1896-1899.

Ogawa, T., A. Shinohara, A. Nabetani, T. Ikeya, X. Yu, E.H. Egelman, and H. Ogawa. 1993b. RecA-like recombination proteins in eukaryotes: Functions and structures of RAD51 genes. Cold Spring Harbor Symp. Quant. Biol. 58: 567-576. 
Terasawa et al.

Ogawa, T., A. Shinohara, and T. Ikeya. 1995. A species-specific interaction of Rad51 and Rad52 proteins. Adv. Biophys. 31: 93-100.

Ostermann, K., A. Lorentz, and H. Schmidt. 1993. The fission yeast rad22 gene, having a function in mating-type switching and repair of DNA damages, encodes a protein homolog to Rad52 of Saccharomyces cerevisiae. Nucleic Acids Res. 21: 5940-5944.

Petes, T., R.E. Malone, and L.S. Symington. 1991. Recombination in yeast. In The molecular and cellular biology of the yeast Saccharomyces (ed. J.R. Broach, J.R. Pringle, and E.W. Jones). Vol. 1, pp. 407-521. Cold Spring Harbor Laboratory Press, Cold Spring Harbor, New York.

Rasmussen, S.W. and P.B. Holm. 1978. Human meiosis II. Chromosome pairing and recombination nodules in human spermatocytes. Carlsberg Res. Commun. 43: 275-327.

Resnick, M.A. 1987. Investigating the genetic control of biochemical events in meiotic recombination. In Meiosis. (ed. P.B. Moens\}, pp. 175-210. Academic Press, New York.

Shinohara, A., H. Ogawa, and T. Ogawa. 1992. Rad51 protein involved in repair and recombination in $S$. cerevisiae is a RecA-like protein. Cell 69: 457-470.

Shinohara, A., H. Ogawa, Y. Matsuda, K. Ikeo, N. Ushio, and T. Ogawa. 1993. Cloning of human, mouse and fission yeast recombination genes homologous to RAD51 and recA. $\mathrm{Na}$ ture Genet. 4: 239-243.

Stack, S.M., L.K. Anderson, and J.D. Sherman. 1989. Chiasmata and recombination nodules in Lilium longiflorum. Genome 32: $486-498$.

Story, R.M., D.K. Bishop, N. Kleckner, and T.A. Steiz. 1993. Structural relationship of bacterial RecA proteins to recombination proteins from bacteriophage $\mathrm{T} 4$ and yeast. Science 249: 1892-1896.

Studier, F.W., A.H. Rosenberg, J.J. Dunn, and J.W. Dubendorff. 1990. Use of T7 RNA polymerase to direct expression of cloned genes. Methods Enzymol. 185: 60-89.

Sung, P. 1994. Catalysis of ATP-dependent homologous DNA pairing and strand exchange by yeast RAD51 protein. Science 265: 1241-1243.

von Wettstein, D., S.W. Rasmussen, and P.B. Holm. 1984. The synaptonemal complex in genetic segregation. Annu. Rev. Genet. 18: $331-413$.

Zickler, D., P.J.F. Moreau, A.D. Huynh, and A.-M. Slezec. 1992. Correlation between pairing initiation sites, recombination nodules and meiotic recombination in Sordaria macrospora. Genetics 132: 135-148. 


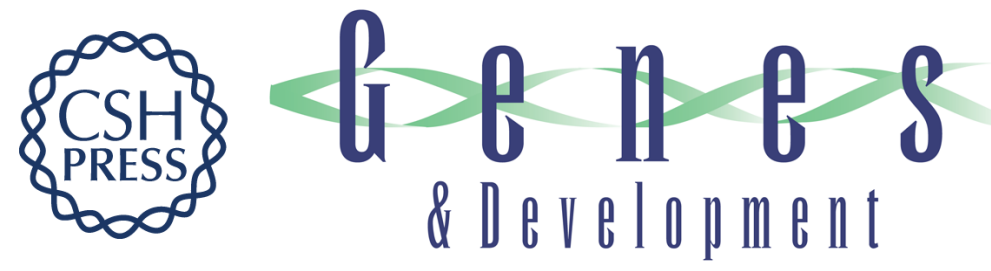

\section{Localization of RecA-like recombination proteins on chromosomes of the lily at various meiotic stages.}

M Terasawa, A Shinohara, Y Hotta, et al.

Genes Dev. 1995, 9:

Access the most recent version at doi:10.1101/gad.9.8.925

References This article cites 34 articles, 9 of which can be accessed free at: http://genesdev.cshlp.org/content/9/8/925.full.html\#ref-list-1

License

Email Alerting

Service

Receive free email alerts when new articles cite this article - sign up in the box at the top right corner of the article or click here.

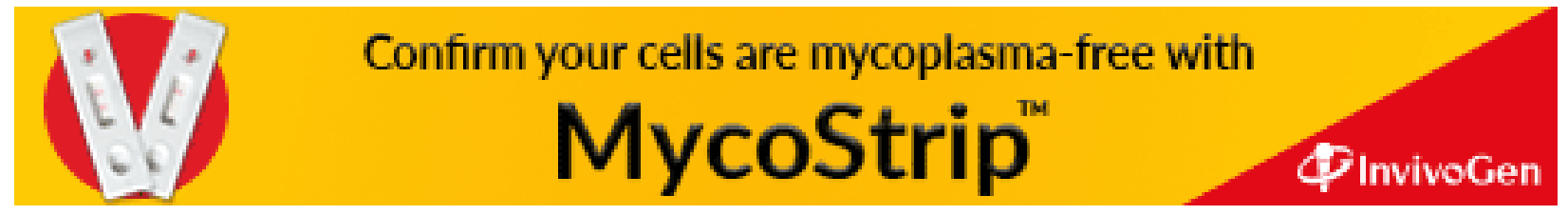

